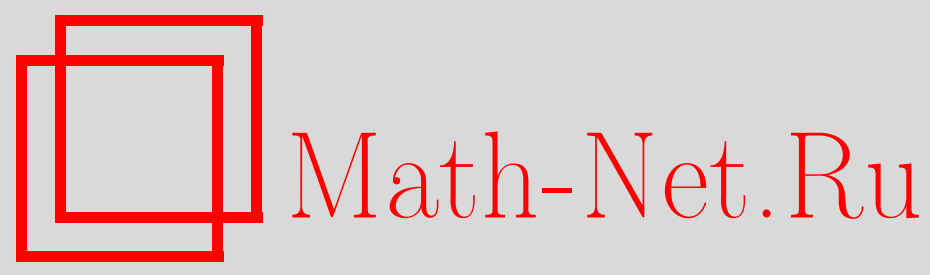

Х. Д. Икрамов, А. М. Назари, О расстоянии до ближайшей матрицы с тройным собственным значением нуль, Матем. заметки, 2003, том 73, выпуск 4, 545-555

DOI: https://doi.org/10.4213/mzm202

Использование Общероссийского математического портала Math-Net.Ru подразумевает, что вы прочитали и согласны с пользовательским соглашением http://www.mathnet.ru/rus/agreement

Параметры загрузки:

IP : 34.227 .88 .159

26 апреля 2023 г., $17: 24: 00$

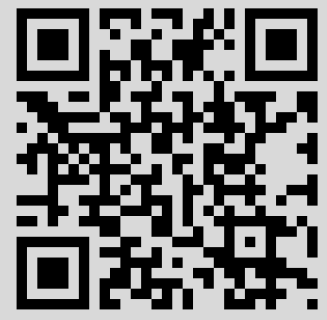




\title{
О РАССТОЯНИИ ДО БЛИЖАЙШЕЙ МАТРИЦЫ С ТРОЙНЫМ СОБСТВЕННЫМ ЗНАЧЕНИЕМ НУЛЬ
}

\author{
Х. Д. Икрамов, А. М. Назари
}

Оценивается спектральное расстояние от $A$ до множества $\mathscr{M}(n \times n)$-матриц, имеющих собственное значение 0 кратности $\geqslant 3$. Если

$$
Q\left(\gamma_{1}, \gamma_{2}, \gamma_{3}\right)=\left(\begin{array}{ccc}
A & \gamma_{1} I_{n} & \gamma_{3} I_{n} \\
0 & A & \gamma_{2} I_{n} \\
0 & 0 & A
\end{array}\right), \quad n \geqslant 3
$$

то

$$
\rho_{2}(A, \mathscr{M}) \geqslant \max _{\gamma_{1}, \gamma_{2} \geqslant 0, \gamma_{3} \in \mathbb{C}} \sigma_{3 n-2}\left(Q\left(\gamma_{1}, \gamma_{2}, \gamma_{3}\right)\right),
$$

где $\sigma_{i}(\cdot)$ есть $i$-е сингулярное число соответствующей матрицы при упорядочении сингулярных чисел по убыванию. При этом, если максимум в правой части достигается в точке $\gamma^{*}=\left(\gamma_{1}^{*}, \gamma_{2}^{*}, \gamma_{3}^{*}\right)$, где $\gamma_{1}^{*} \gamma_{2}^{*} \neq 0$, то, в действительности, имеет место точное равенство

$$
\rho_{2}(A, \mathscr{M})=\sigma_{3 n-2}\left(Q\left(\gamma_{1}^{*}, \gamma_{2}^{*}, \gamma_{3}^{*}\right)\right)
$$

Этот результат можно рассматривать как обобщение формулы А.Н. Малышева для спектрального расстояния от $A$ до множества матриц с кратным собственным значением нуль.

Библиограффия: 3 названия.

1. Введение. Пусть $A$-комплексная $(n \times n)$-матрица, $\mathscr{L}$-множество $(n \times n)$-матриц с кратным собственньм значением 0. В недавней статье А. Н. Мальшева [1] получена следующая формула для спектрального расстояния от $A$ до $\mathscr{L}$ :

$$
\rho_{2}(A, \mathscr{L})=\min _{L \in \mathscr{L}}\|A-L\|_{2}=\max _{\gamma \geqslant 0} \sigma_{2 n-1}\left(P_{\lambda}(\gamma)\right) .
$$

Здесь

$$
P_{\lambda}(\gamma)=\left(\begin{array}{cc}
A & \gamma I_{n} \\
0 & A
\end{array}\right)
$$

a $\sigma_{i}(\cdot)$ обозначает $i$-е сингулярное число соответствуюшей матрищы. Предполагается, что сингулярные числа всякой матрищы упорядочены по убьванию.

Цель настоящего сообщения - указать аналогичную формулу для спектрального расстояния от $A$ до множества $\mathscr{M}$ матриц, имеющих собственное значение 0 кратности $\geqslant 3$. Положим

$$
Q\left(\gamma_{1}, \gamma_{2}, \gamma_{3}\right)=\left(\begin{array}{ccc}
A & \gamma_{1} I_{n} & \gamma_{3} I_{n} \\
0 & A & \gamma_{2} I_{n} \\
0 & 0 & A
\end{array}\right), \quad n \geqslant 3 .
$$


Тогда

$$
\rho_{2}(A, \mathscr{M}) \geqslant \max _{\gamma_{1}, \gamma_{2} \geqslant 0, \gamma_{3} \in \mathbb{C}} \sigma_{3 n-2}\left(Q\left(\gamma_{1}, \gamma_{2}, \gamma_{3}\right)\right)
$$

При этом, если максимум в правой части достигается в точке $\gamma^{*}=\left(\gamma_{1}^{*}, \gamma_{2}^{*}, \gamma_{3}^{*}\right)$, где $\gamma_{1}^{*} \gamma_{2}^{*} \neq 0$, то (4) преврашается в точное равенство

$$
\rho_{2}(A, \mathscr{M})=\sigma_{3 n-2}\left(Q\left(\gamma_{1}^{*}, \gamma_{2}^{*}, \gamma_{3}^{*}\right)\right)
$$

Доказательство этого утверждения проводится в п. 2-5. С определенными усложнениями оно следует схеме, примененной Мальшевым в [1]. Центральным моментом доказательства является лемма 6, представляющая собой “многопараметрический” вариант одноименной леммы Мальшева.

2. Нижняя оценка для $\rho_{2}(A, \mathscr{M})$. В этом пункте будет доказано неравенство (4). Доказательство использует две леммы, первая из которых хорошо известна.

Лемма 1. Матрииа В порядка $n$ имеет собственное значение 0 кратности $\geqslant 3$ тогда и только тогда, когда

$$
\operatorname{rank} B^{3} \leqslant n-3
$$

ЛЕмма 2. Если выполнено условие (6), то матрица

$$
Q_{B}=\left(\begin{array}{ccc}
B & \gamma_{1} I_{n} & \gamma_{3} I_{n} \\
0 & B & \gamma_{2} I_{n} \\
0 & 0 & B
\end{array}\right)
$$

имеет ранг $\leqslant 3 n-3$, каковы бы ни били $\gamma_{1}, \gamma_{2} u \gamma_{3}$.

ДокАЗАТЕЛЬСТво. Прежде всего, заметим, что неравенство (6) возможно лишш при условии, что матрица $B$ вырождена и

$$
\operatorname{rank} B^{2} \leqslant n-2
$$

При $\gamma_{1}=\gamma_{2}=\gamma_{3}=0$ утверждение леммы очевидно. Если $\gamma_{1}=\gamma_{2}=0, \gamma_{3} \neq 0$, то матрица (7) эквивалентна каждой из матриц

$$
\left(\begin{array}{ccc}
B & \gamma_{3} I_{n} & 0 \\
0 & B & 0 \\
0 & 0 & B
\end{array}\right) \sim\left(\begin{array}{ccc}
B & \gamma_{3} I_{n} & 0 \\
-\left(1 / \gamma_{3}\right) B^{2} & 0 & 0 \\
0 & 0 & B
\end{array}\right) \sim\left(\begin{array}{ccc}
0 & \gamma_{3} I_{n} & 0 \\
-\left(1 / \gamma_{3}\right) B^{2} & 0 & 0 \\
0 & 0 & B
\end{array}\right) \sim I_{n} \oplus B^{2} \oplus B
$$

Эквивалентность двух матриц понимается здесь как возможность перевести любую из них в другую посредством элементарных преобразований строк и столбцов. Из (8) следует, что ранг матрицы $(9) \leqslant 3 n-3$.

Предположим теперь, что $\gamma_{1} \gamma_{2}=0$, но одно из этих чисел ненулевое; пусть, для определенности, $\gamma_{1} \neq 0$. Тогда

$$
\left(\begin{array}{ccc}
B & \gamma_{1} I_{n} & \gamma_{3} I_{n} \\
0 & B & 0 \\
0 & 0 & B
\end{array}\right) \sim\left(\begin{array}{ccc}
B & \gamma_{1} I_{n} & 0 \\
0 & B & -\left(\gamma_{3} / \gamma_{1}\right) B \\
0 & 0 & B
\end{array}\right) \sim\left(\begin{array}{ccc}
B & \gamma_{1} I_{n} & 0 \\
0 & B & 0 \\
0 & 0 & B
\end{array}\right) \sim I_{n} \oplus B^{2} \oplus B
$$


Таким образом, лемма справедлива и в этом случае.

Пусть, наконец, $\gamma_{1} \neq 0$ и $\gamma_{2} \neq 0$. Имеем

$$
\begin{aligned}
\left(\begin{array}{ccc}
B & \gamma_{1} I_{n} & \gamma_{3} I_{n} \\
0 & B & \gamma_{2} B \\
0 & 0 & B
\end{array}\right) & \sim\left(\begin{array}{ccc}
B & \gamma_{1} I_{n} & 0 \\
0 & B & \gamma_{2} I_{n}-\left(\gamma_{3} / \gamma_{1}\right) B \\
0 & 0 & B
\end{array}\right) \sim\left(\begin{array}{ccc}
B & \gamma_{1} I_{n} & 0 \\
0 & B & \gamma_{2} I_{n} \\
0 & 0 & B
\end{array}\right) \\
& \sim\left(\begin{array}{cccc}
0 & \gamma_{1} I_{n} & 0 \\
-\left(1 / \gamma_{1}\right) B^{2} & 0 & \gamma_{2} I_{n} \\
0 & 0 & B
\end{array}\right) \sim\left(\begin{array}{ccc}
0 & \gamma_{1} I_{n} & 0 \\
0 & 0 & \gamma_{2} I_{n} \\
\left(1 /\left(\gamma_{1} \gamma_{2}\right)\right) B^{3} & 0 & 0
\end{array}\right) \\
& \sim I_{n} \oplus I_{n} \oplus B^{3} .
\end{aligned}
$$

Теперь утверждение леммы следует из неравенства (6).

Перейдем к доказательству неравенства (4). Пусть $\Delta$ - матрица минимальной спектральной нормы такая, что $B=A+\Delta \in \mathscr{M}$. Согласно лемме 2 ранг матрицы $Q_{B}$ $($ см. $(7))$ не превосходит $3 n-3$, поэтому сингулярное число $\sigma_{3 n-2}\left(Q_{B}\right)$ равно нулю. Представим $Q_{B}$ в виде

$$
Q_{B}=Q\left(\gamma_{1}, \gamma_{2}, \gamma_{3}\right)+\left(\begin{array}{ccc}
\Delta & 0 & 0 \\
0 & \Delta & 0 \\
0 & 0 & \Delta
\end{array}\right)=Q\left(\gamma_{1}, \gamma_{2}, \gamma_{3}\right)+D
$$

Учитывая, что $\|D\|_{2}=\|\Delta\|_{2}$, и применяя неравенства Вейля для сингулярных чисел (см., например, [2, следствие 5.1]), вьводим из представления (12) и равенства $\sigma_{3 n-2}\left(Q_{B}\right)=0$ оценку

$$
\|D\|_{2} \geqslant \sigma_{3 n-2}\left(Q\left(\gamma_{1}, \gamma_{2}, \gamma_{3}\right)\right)
$$

Ввиду произвольности $\gamma$, получаем требуемое неравенство (4).

3. Свойства сингулярных векторов матрицы $Q\left(\gamma_{1}, \gamma_{2}, \gamma_{3}\right)$. Пусть

$$
\sigma^{*}=\max _{\gamma_{1}, \gamma_{2} \geqslant 0, \gamma_{3} \in \mathbb{C}} \sigma_{3 n-2}\left(Q\left(\gamma_{1}, \gamma_{2}, \gamma_{3}\right)\right) \text {. }
$$

Если $\sigma^{*}=0$, то, в частности, $\sigma_{3 n-2}(Q(1,1,0))=0$. Выкладки, аналогичные тем, что проводились в предыдущем пункте, приводят к соотношению

$$
Q(1,1,0) \sim I_{n} \oplus I_{n} \oplus A^{3}
$$

откуда следует, что $\operatorname{rank} A^{3} \leqslant n-3$ и, стало быть, $A \in \mathscr{M}$. Таким образом, при $\sigma^{*}=0$ формула (5) вьполняется. Это позволяет нам в дальнейшем считать, что $\sigma^{*}>0$.

Рассуждениями, начинающимися здесь и продолжающимися до конца п. 5, мы покажем (при ограничениях, указанных во введении) существование матрицы $\Delta$ такой, что

$$
\|\Delta\|_{2}=\sigma^{*}
$$

и

$$
A+\Delta \in \mathscr{M}
$$


Этим будет доказана формула (5).

Построение матрицы $\Delta$ опирается на свойства сингулярных векторов матрицы $Q\left(\gamma_{1}\right.$, $\left.\gamma_{2}, \gamma_{3}\right)$ как в произвольной точке

$$
\gamma=\left(\gamma_{1}, \gamma_{2}, \gamma_{3}\right)
$$

так и в точке $\gamma=\gamma^{*}$, реализующей локальный экстремум функции

$$
f(\gamma)=\sigma_{3 n-2}\left(Q\left(\gamma_{1}, \gamma_{2}, \gamma_{3}\right)\right)
$$

В настоящем пункте перечисляются свойства, справедливые для всех $\gamma$.

Пусть $\sigma$ - сингулярное число матрицы $Q(\gamma)=Q\left(\gamma_{1}, \gamma_{2}, \gamma_{3}\right)$, а $u$ и $v$ - соответственно правый и левый сингулярные векторы, ассоциированные с $\sigma$. Это значит, что

$$
Q u=\sigma v, \quad Q^{*} v=\sigma u \text {. }
$$

Без ограничения обшности можно считать, что $\|u\|_{2}=\|v\|_{2}=1$.

Представим $u$ и $v$ в блочном виде

$$
u=\left(\begin{array}{l}
u_{1} \\
u_{2} \\
u_{3}
\end{array}\right), \quad v=\left(\begin{array}{l}
v_{1} \\
v_{2} \\
v_{3}
\end{array}\right)
$$

где каждый из подвекторов $u_{i}$ и $v_{j}$ имеет размерность $n$.

Равенства (17) приводят к соотношениям

$$
\begin{gathered}
A u_{1}+\gamma_{1} u_{2}+\gamma_{3} u_{3}=\sigma v_{1}, \\
A u_{2}+\gamma_{2} u_{3}=\sigma v_{2}, \\
A u_{3}=\sigma v_{3}, \\
A^{*} v_{1}=\sigma u_{1}, \\
A^{*} v_{2}+\gamma_{1} v_{1}=\sigma u_{2}, \\
A^{*} v_{3}+\bar{\gamma}_{3} v_{1}+\gamma_{2} v_{2}=\sigma u_{3} .
\end{gathered}
$$

ЛЕмма 3. Если $\sigma>0, m o$

$$
v_{1}^{*} v_{3}=u_{1}^{*} u_{3}
$$

ДокАЗАТЕЛЬСтво. Умножим (20) скалярно на $v_{1}$, а $(21)$ на $u_{3}$. Беря сопряжение от второго из полученных соотношений и вычитая его из первого, приходим к равенству

$$
\sigma\left(v_{1}^{*} v_{3}-u_{1}^{*} u_{3}\right)=0
$$

откуда следует (24).

Лемма 4. Для любых $\delta_{1}, \delta_{2}, \delta_{3} \in \mathbb{R}$ справедливо равенство

$$
\begin{aligned}
& \gamma_{1}\left(\delta_{1}-\delta_{2}\right) v_{1}^{*} u_{2}+\gamma_{2}\left(\delta_{2}-\delta_{3}\right) v_{2}^{*} u_{3}+\gamma_{3}\left(\delta_{1}-\delta_{3}\right) v_{1}^{*} u_{3} \\
& =\sigma\left[\delta_{1}\left(v_{1}^{*} v_{1}-u_{1}^{*} u_{1}\right)+\delta_{2}\left(v_{2}^{*} v_{2}-u_{2}^{*} u_{2}\right)+\delta_{3}\left(v_{3}^{*} v_{3}-u_{3}^{*} u_{3}\right)\right] .
\end{aligned}
$$


ДокАЗАтЕльство. Умножим равенства (18)-(20) скалярно соответственно на $\delta_{1} v_{1}$, $\delta_{2} v_{2}, \delta_{3} v_{3}$. Затем умножим (21)-(23) соответственно на $\delta_{1} u_{1}, \delta_{2} u_{2}, \delta_{3} u_{3}$. Возьмем сумму первых трех полученных соотношений и вычтем из нее результат комплексного сопряжения, примененного к сумме трех остальных соотношений. Это приводит к равенствy (26).

ЛЕмма 5. Справедливы соотношения

$$
\begin{aligned}
& \gamma_{2} v_{1}^{*} u_{3}=\sigma\left(v_{1}^{*} v_{2}-u_{1}^{*} u_{2}\right) \\
& \gamma_{1} v_{1}^{*} u_{3}=\sigma\left(u_{2}^{*} u_{3}-v_{2}^{*} v_{3}\right)
\end{aligned}
$$

ДокАЗАТЕЛЬСТво. Чтобы получить (27), умножим скалярно (19) на $v_{1}$, а (21) на $u_{2}$, а затем вычтем из первого соотношения сопряженное ко второму. Аналогичныевыкладки, где (20) умножается на $v_{2}$, а $(22)$ на $u_{3}$, приводят к (28).

4. Дополнительные свойства в точке локального экстремума. Пусть в точке

$$
\gamma^{*}=\left(\gamma_{1}^{*}, \gamma_{2}^{*}, \gamma_{3}^{*}\right)
$$

реализуется локальный экстремум $\sigma^{*}$ функции $f(\gamma)$. Напомним, что $\gamma_{1}$ и $\gamma_{2}$ вещественны, а $\gamma_{3}=\gamma_{3 R}+i \gamma_{3 I}-$ комплексная переменная. Удобно положить

$$
\gamma_{1}=\xi_{1}, \quad \gamma_{2}=\xi_{2}, \quad \gamma_{3 R}=\xi_{3}, \quad \gamma_{3 I}=\xi_{4}
$$

и рассматривать $f$ как функцию четырех вешественных переменных $\xi_{1}, \ldots, \xi_{4}$. Сингулярные векторы матрищы $Q$, относящиеся к числу $\sigma^{*}$ в точке локального экстремума

$$
\xi^{*}=\left(\xi_{1}^{*}, \xi_{2}^{*}, \xi_{3}^{*}, \xi_{4}^{*}\right)
$$

помимо свойств, выражаемьх леммами $3-5$, подчиняются соотношениям, заключенным в следующей лемме.

ЛЕмма 6. Если точка $\xi^{*}$ (см. (29)) реализует локальный әкстремум $\sigma^{*}>0$ функиии $f$, то найдутся ассоциированные с $\sigma^{*}$ правый и левый сингулярные векторы $и$ u $v$ mакие, что

$$
\begin{aligned}
& \operatorname{Re}\left(v^{*} \frac{\partial Q}{\partial \xi_{1}}\left(\xi^{*}\right) u\right)=0 \\
& \operatorname{Re}\left(v^{*} \frac{\partial Q}{\partial \xi_{2}}\left(\xi^{*}\right) u\right)=0 \\
& \operatorname{Re}\left(v^{*} \frac{\partial Q}{\partial \xi_{3}}\left(\xi^{*}\right) u\right)=0 \\
& \operatorname{Re}\left(v^{*} \frac{\partial Q}{\partial \xi_{4}}\left(\xi^{*}\right) u\right)=0
\end{aligned}
$$


ДоКАЗАТЕЛЬСТво. Если $\sigma^{*}-$ простое сингулярное число матрищы $Q\left(\xi^{*}\right)$, то

$$
f(\xi)=\sigma_{3 n-2}(Q(\xi))
$$

является (вещественной) аналитической функцией переменных $\xi_{1}, \ldots, \xi_{4}$ в некоторой окрестности точки $\xi^{*}$ и соотношения (30)-(33) суть попросту необходимые условия экстремума, записанные с помощью формулы дифференцирования сингулярного числа из [3, лемма 4].

Предположим теперь, что $\sigma^{*}-$ кратное сингулярное число. В некоторой окрестности точки $\xi^{*}$ рассмотрим функции

$$
\sigma_{-} \equiv \sigma_{3 n-2}(\xi), \quad \sigma_{+} \equiv \sigma_{3 n-3}(\xi)
$$

Они не дифференцируемы в $\xi^{*}$ вследствие наложенного на них условия упорядоченности $\sigma_{3 n-2} \leqslant \sigma_{3 n-3}$. Однако если отказаться от этого условия, то в окрестности точки $\xi^{*}$ определены (вещественные) аналитические функции $\tilde{\sigma}_{-}(\xi)$ и $\tilde{\sigma}_{+}(\xi)$, также имеющие смысл сингулярных чисел, причем

$$
\begin{aligned}
& \sigma_{-}(\xi)=\min \left\{\tilde{\sigma}_{-}(\xi), \tilde{\sigma}_{+}(\xi)\right\} \\
& \sigma_{+}(\xi)=\max \left\{\tilde{\sigma}_{-}(\xi), \tilde{\sigma}_{+}(\xi)\right\}
\end{aligned}
$$

Пусть $u_{-}(\xi), v_{-}(\xi)$ и $u_{+}(\xi), v_{+}(\xi)$ суть пары сингулярных векторов, ассоциированных соответственно с $\tilde{\sigma}_{-}(\xi)$ и $\tilde{\sigma}_{+}(\xi)$. Уравнение

$$
F\left(\xi_{1}, \xi_{2}, \xi_{3}, \xi_{4}\right) \equiv \tilde{\sigma}_{+}\left(\xi_{1}, \xi_{2}, \xi_{3}, \xi_{4}\right)-\tilde{\sigma}_{-}\left(\xi_{1}, \xi_{2}, \xi_{3}, \xi_{4}\right)=0
$$

задает трехмерное (вещественное) аналитическое многообразие, содержащее точку $\xi^{*}$. Выполним линейную замену переменных

$$
\xi-\xi^{*}=S \eta, \quad \operatorname{det} S \neq 0,
$$

так, чтобы в переменных $\eta_{1}, \ldots, \eta_{4}$ касательная гиперплоскость к многообразию (37) описьвалась уравнением

$$
\eta_{4}=0 \text {. }
$$

Пусть $l$ - произвольньй ненулевой вектор, принадлежащий этой гиперплоскости. Тогда производная функции $F$ в точке 0 по направлению $l$ равна нулю, т.е.

$$
\frac{\partial \tilde{\sigma}_{+}}{\partial l}(0)=\frac{\partial \tilde{\sigma}_{-}}{\partial l}(0) .
$$

В действительности, эти производные тоже равны нулю. Если допустить противное, например, что они положительны, то при смещении из нуля в направлении $l$ обе функции $\tilde{\sigma}_{+}$и $\tilde{\sigma}_{-}$возрастают, а при смещении в противоположном направлении убьвают. Таким же образом ведет себя функция (35), что противоречит исходному предположению: точка $\xi^{*}$ есть локальный экстремум функции $f(\xi)$.

Равенства

$$
\frac{\partial \tilde{\sigma}_{+}}{\partial l}(0)=0, \quad \frac{\partial \tilde{\sigma}_{-}}{\partial l}(0)=0
$$


означают, что

$$
\operatorname{Re}\left(v_{+}^{*}(0) \frac{\partial Q}{\partial l} u_{+}(0)\right)=0
$$

и

$$
\operatorname{Re}\left(v_{-}^{*}(0) \frac{\partial Q}{\partial l} u_{-}(0)\right)=0 .
$$

Пусть $u$ - произвольный вектор из вещественной линейной оболочки векторов $u_{+}(0)$ и $u_{-}(0)$ :

$$
u=\alpha u_{+}(0)+\beta u_{-}(0), \quad \alpha, \beta \in \mathbb{R} .
$$

Таким образом, $u$ является правым сингулярным вектором, относящимся к $\sigma^{*}$. Пусть $v$ - соответствующий левьй сингулярный вектор, т.е. $Q(0) u=\sigma^{*} v$ и

$$
v=\alpha v_{+}(0)+\beta v_{-}(0) .
$$

Мы утверждаем, что

$$
\operatorname{Re}\left(v^{*} \frac{\partial Q}{\partial l} u\right)=0
$$

Предположим противное, т.е.

$$
\begin{aligned}
\operatorname{Re}\left(v^{*} \frac{\partial Q}{\partial l} u\right)= & \alpha^{2} \operatorname{Re}\left(v_{+}^{*}(0) \frac{\partial Q}{\partial l} u_{+}(0)\right)+\beta^{2} \operatorname{Re}\left(v_{-}^{*}(0) \frac{\partial Q}{\partial l} u_{-}(0)\right) \\
& +\alpha \beta \operatorname{Re}\left(v_{+}^{*}(0) \frac{\partial Q}{\partial l} u_{-}(0)+v_{-}^{*}(0) \frac{\partial Q}{\partial l} u_{+}(0)\right) \neq 0
\end{aligned}
$$

т.е. в силу $(40)$ и $(41)$

$$
\operatorname{Re}\left\{\left(v_{+}^{*}(0) \frac{\partial Q}{\partial l} u_{-}(0)\right)+\overline{\left(u_{+}^{*}(0) \frac{\partial Q^{*}}{\partial l} v_{-}(0)\right)}\right\} \neq 0 .
$$

Чтобы воспользоваться классическими результатами Реллиха о возмущениях собственных значений эрмитовых операторов, рассмотрим поведение кратного собственного значения $\left(\sigma^{*}\right)^{2}$ матрищы $Q^{*}\left(\gamma^{*}\right) Q\left(\gamma^{*}\right)$ при малых возмущениях $\gamma$. Это поведение в переменных $\eta_{1}, \ldots, \eta_{4}$ определяется собственными значениями $(2 \times 2)$-матрицы

$$
\begin{aligned}
& \left(\begin{array}{l}
u_{+}^{*}(0) \\
u_{-}^{*}(0)
\end{array}\right)\left(Q^{*}(0) \frac{\partial Q}{\partial l}+\frac{\partial Q^{*}}{\partial l} Q(0)\right)\left(u_{+}(0) u_{-}(0)\right) \\
& \quad=\sigma^{*}\left(\begin{array}{cc}
2 \operatorname{Re}\left(v_{+}^{*}(0) \frac{\partial Q}{\partial l} u_{+}(0)\right) & a \\
\bar{a} & \operatorname{Re}\left(v_{-}^{*}(0) \frac{\partial Q}{\partial l} u_{-}(0)\right)
\end{array}\right) \\
& =\sigma^{*}\left(\begin{array}{ll}
0 & a \\
\bar{a} & 0
\end{array}\right) .
\end{aligned}
$$

В этих выкладках использованы соотношения

$$
Q(0) u_{+}(0)=\sigma^{*} v_{+}(0), \quad Q(0) u_{-}(0)=\sigma^{*} v_{-}(0)
$$


и равенства $(40)$ и (41). Что касается элемента $(1,2)$, то

$$
a=v_{+}^{*}(0) \frac{\partial Q}{\partial l} u_{-}(0)+u_{+}^{*}(0) \frac{\partial Q}{\partial l} v_{-}(0) .
$$

В силу (45) $a \neq 0$ и матрица (46) имеет два ненулевых собственных значения $\sigma^{*}|a|$ и $-\sigma^{*}|a|$. Эти числа имеют смысл производных в нуле для двух аналитических собственных значений матрицы $Q^{*}(\eta) Q(\eta)$, порождаемых расщеплением кратного собственного значения $\left(\sigma^{*}\right)^{2}$ при смещении из нуля в направлении $l$. То обстоятельство, что производные не равны нулю, противоречит равенствам (39). Это противоречие доказывает (44).

Вернемся к сингулярным числам матрицы $Q(\eta)$ и рассмотрим их поведение при смешении из нуля в направлении $\eta_{4}$. При таком смещении мы попадаем в область, где

$$
\tilde{\sigma}_{+}(\eta) \geqslant \sigma^{*} \geqslant \tilde{\sigma}_{-}(\eta)
$$

либо

$$
\tilde{\sigma}_{-}(\eta) \leqslant \sigma^{*} \leqslant \tilde{\sigma}_{+}(\eta)
$$

Это означает, что

или

$$
\frac{\partial \tilde{\sigma}_{+}}{\partial \eta_{4}}(0) \frac{\partial \tilde{\sigma}_{-}}{\partial \eta_{4}}(0) \leqslant 0
$$

$$
\operatorname{Re}\left(v_{+}^{*}(0) \frac{\partial Q}{\partial \eta_{4}} u_{+}(0)\right) \operatorname{Re}\left(v_{-}^{*}(0) \frac{\partial Q}{\partial \eta_{4}} u_{-}(0)\right) \leqslant 0 .
$$

Далее используем идею, заимствованную из леммы 5 в [1]. Полагая в (42) и (43) $0 \leqslant \alpha \leqslant 1, \beta=\sqrt{1-\alpha^{2}}$, определим непрерьвную функцию от $\alpha$ формулой

$$
\phi(\alpha)=\operatorname{Re}\left(v^{*} \frac{\partial Q}{\partial \eta_{4}} u\right)
$$

Если оба числа

$$
\phi(0)=\operatorname{Re}\left(v_{+}^{*}(0) \frac{\partial Q}{\partial \eta_{4}} u_{+}(0)\right)
$$

и

$$
\phi(1)=\operatorname{Re}\left(v_{-}^{*}(0) \frac{\partial Q}{\partial \eta_{4}} u_{-}(0)\right)
$$

отличны от нуля, то согласно (47) они противоположны по знаку. Тогда найдется $\alpha_{0} \in$ $(0,1)$ такое, что

$$
\phi\left(\alpha_{0}\right)=\operatorname{Re}\left(v^{*} \frac{\partial Q}{\partial \eta_{4}} u\right)=0
$$

Подведем итог проведенным рассуждениям. Мы показали, что найдется пара сингулярных векторов $u$ и $v$ такая, что вьполнено (48). В то же время эта пара удовлетворяет соотношению (44), каково бы ни было направление $l$, касательное в нуле к многообразию (37). Отсюда следует, что

$$
\operatorname{Re}\left(v^{*} \frac{\partial Q}{\partial l} u\right)=0
$$

для любого ненулевого вектора $l \in \mathbb{C}^{4}$. Возвращаясь к переменным $\xi_{1}, \ldots, \xi_{4}$ и используя (49) для смещений вдоль координатных осей, получаем соотношения (30)-(33). Лемма доказана. 
ЗАмЕЧАниЕ. Соотношения (30)-(33) равносильны соотношениям

$$
\begin{aligned}
\operatorname{Re}\left(v_{1}^{*} u_{2}\right) & =0, \\
\operatorname{Re}\left(v_{2}^{*} u_{3}\right) & =0, \\
v_{1}^{*} u_{3} & =0 .
\end{aligned}
$$

Это следует из формул

$$
\begin{array}{ccc}
\frac{\partial Q}{\partial \xi_{1}}= & \frac{\partial Q}{\partial \gamma_{1}}=\left(\begin{array}{ccc}
0 & I & 0 \\
0 & 0 & 0 \\
0 & 0 & 0
\end{array}\right), & \frac{\partial Q}{\partial \xi_{2}}=\frac{\partial Q}{\partial \gamma_{2}}=\left(\begin{array}{ccc}
0 & 0 & 0 \\
0 & 0 & I \\
0 & 0 & 0
\end{array}\right), \\
\frac{\partial Q}{\partial \xi_{3}}=\left(\begin{array}{ccc}
0 & 0 & I \\
0 & 0 & 0 \\
0 & 0 & 0
\end{array}\right), & \frac{\partial Q}{\partial \xi_{4}}=i\left(\begin{array}{ccc}
0 & 0 & I \\
0 & 0 & 0 \\
0 & 0 & 0
\end{array}\right) .
\end{array}
$$

СЛЕДСТВИЕ. Составим $(n \times 3)$-матрицы

$$
U=\left(u_{1} u_{2} u_{3}\right) \quad u \quad V=\left(v_{1} v_{2} v_{3}\right)
$$

из подвекторов сингулярных векторов и и v, описываемых в лемме 6. Тогда

$$
V^{*} V=U^{*} U
$$

ДокАЗАТЕЛЬСТво. Поскольку $\sigma^{*}>0$, из (27) и (28) находим

$$
\begin{aligned}
& v_{1}^{*} v_{2}=u_{1}^{*} u_{2}, \\
& v_{2}^{*} v_{3}=u_{2}^{*} u_{3} .
\end{aligned}
$$

В силу (50)-(52) левая часть соотношения (26) имеет нулевую вещественную часть, откуда

$$
\delta_{1}\left(v_{1}^{*} v_{1}-u_{1}^{*} u_{1}\right)+\delta_{2}\left(v_{2}^{*} v_{2}-u_{2}^{*} u_{2}\right)+\delta_{3}\left(v_{3}^{*} v_{3}-u_{3}^{*} u_{3}\right)=0,
$$

каковы бы ни были числа $\delta_{1}, \delta_{2}$ и $\delta_{3}$. Это означает, что

$$
\begin{aligned}
& v_{1}^{*} v_{1}=u_{1}^{*} u_{1}, \\
& v_{2}^{*} v_{2}=u_{2}^{*} u_{2}, \\
& v_{3}^{*} v_{3}=u_{3}^{*} u_{3} .
\end{aligned}
$$

Наконец, вьполняется лемма 3. Матричное равенство (54) есть точньй эквивалент соотношения (24) этой леммы, объединенного с соотношениями (55)-(59). 
5. Построение минимального возмущения $\Delta$. Как уже было сказано, построение матрицы $\Delta$, удовлетворяющей условиям (14) и (15), использует сингулярные векторы $u$ и $v$, исследованные в двух предыдущих пунктах статьи. Эти векторы относятся к сингулярному числу $\sigma^{*}$, являющемуся значением функции $f(\gamma)($ см. $(16))$ в точке локального экстремума

$$
\gamma^{*}=\left(\gamma_{1}^{*}, \gamma_{2}^{*}, \gamma_{3}^{*}\right)
$$

Наше построение предполагает, что

$$
\gamma_{1}^{*} \neq 0, \quad \gamma_{2}^{*} \neq 0
$$

Покажем, что в этом случае столбцы каждой из матриц (53) линейно независимы. Если допустить, что $u_{3}=0$, то равенства (18) и (19) редуцируются к виду

$$
\begin{aligned}
A u_{1}+\gamma_{1}^{*} u_{2} & =\sigma^{*} v_{1} \\
A u_{2} & =\sigma^{*} v_{2} .
\end{aligned}
$$

Вместе с (21) и (22) они составляют соотношения (6) и (7) из статьи Мальшева. Далее применимы рассуждения из раздела 5.1 этой статьи, показьвающие, что векторы $u_{1}$ и $u_{2}$ (следовательно, $v_{1}$ и $v_{2}$ ) линейно независимы. Между тем, равенство (23) дает

$$
\bar{\gamma}_{3}^{*} v_{1}+\gamma_{2}^{*} v_{2}=0
$$

где $\gamma_{2}^{*} \neq 0$. Полученное противоречие доказьвает, что $u_{3} \neq 0$.

Предположим, что

$$
a u_{1}+b u_{2}+c u_{3}=0
$$

Тогда в силу (54)

$$
a v_{1}+b v_{2}+c v_{3}=0
$$

Если в соотношении

$$
a A u_{1}+b A u_{2}+c A u_{3}=0,
$$

производном от (60), заменить векторы $A u_{1}, A u_{2}$ и $A u_{3}$ их выражениями, вытекающими из (18)-(20), а затем вычесть из полученного равенства умноженное на $\sigma^{*}$ равенство (61), то получим

$$
\gamma_{1}^{*} a u_{2}+\left(\gamma_{3}^{*} a+\gamma_{2}^{*} b\right) u_{3}=0
$$

Отсюда

$$
\gamma_{1}^{*} a v_{2}+\left(\gamma_{3}^{*} a+\gamma_{2}^{*} b\right) v_{3}=0
$$

Опять применяя матрицу $A$ к $(63)$, используя (19) и вычитая (64), умноженное на $\sigma^{*}$, находим

$$
\left(\gamma_{1}^{*} \gamma_{2}^{*} a\right) u_{3}=0
$$

откуда $a=0$. Теперь из (63) имеем $b=0$, а из $(60)-c=0$. Итак, векторы $u_{1}, u_{2}$ и $u_{3}$ (следовательно, $v_{1}, v_{2}$ и $v_{3}$ ) линейно независимы.

Снова как в разделе 5.1 из [1], определим матрицу $\Delta$ формулой

$$
\Delta=-\sigma^{*} V U^{+}
$$


Из (54) легко следует (14). Кроме того,

$$
\Delta U=-\sigma^{*} V\left(U^{+} U\right)=-\sigma^{*} V
$$

Отсюда

$$
(A+\Delta) u_{3}=A u_{3}-\sigma^{*} v_{3}=0, \quad(A+\Delta) u_{2}=A u_{2}-\sigma^{*} v_{2}=-\gamma_{2}^{*} u_{3}
$$

и

$$
(A+\Delta)^{2} u_{2}=-\gamma_{2}^{*}(A+\Delta) u_{3}=0
$$

Наконец,

$$
(A+\Delta) u_{1}=A u_{1}-\sigma^{*} v_{1}=-\gamma_{1}^{*} u_{2}-\gamma_{3}^{*} u_{3}
$$

и

$$
(A+\Delta)^{3} u_{1}=-\gamma_{1}^{*}(A+\Delta)^{2} u_{2}-\gamma_{3}^{*}(A+\Delta)^{2} u_{3}=0 .
$$

Итак, матрица $(A+\Delta)^{3}$ имеет, по меньшей мере, трехмерное ядро, а потому $A+\Delta \in \mathscr{M}$.

Отметим, в заключение, что в настоящее время нам не вполне ясна ситуация в тех случаях, когда в точке локального экстремума $\gamma^{*}=\left(\gamma_{1}^{*}, \gamma_{2}^{*}, \gamma_{3}^{*}\right)$ хотя бы одна из координат $\gamma_{1}^{*}, \gamma_{2}^{*}$ равна нулю. Пользуясь приемами из [1], можно и здесь построить матрицу $\Delta \mathrm{c}$ нормой $\|\Delta\|_{2}=\sigma^{*}$ такую, что $A+\Delta$ имеет двойное собственное значение нуль. Остается открытым вопрос: можно ли дополнительным возмущением превратить это двойное собственное значение в тройное, сохранив норму $\sigma^{*}$ суммарного возмущения.

\section{СПИСОК ЦИТИРОВАННОЙ ЛИТЕРАТУРЫ}

[1] Malyshev A. N. A formula for the 2-norm distance from a matrix to the set of matrices with multiple eigenvalues // Numer. Math. 1999. V. 83. P. 443-454.

[2] Деммель Дж. Вычислительная линейная алгебра. Теория и приложения. М.: Мир, 2001.

[3] Sun J.-G. A note on simple non-zero singular values // J. Comput. Math. 1988. V. 6. P. 259-266. 